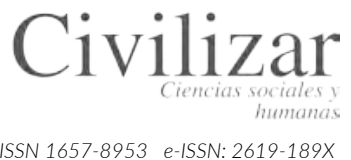

ISSN 1657-8953 e-ISSN: 2619-189X

\title{
La Hermenéutica y la Fenomenología en la Investigación en Ciencias Humanas y Sociales
}

\author{
The Hermeneutics and Phenomenology in Research in Human \\ and Social Sciences \\ John Jairo Pérez Vargas ${ }^{\varsigma}$ \\ Johan Andrés Nieto Bravo ${ }^{\ddagger}$ \\ Juan Esteban Santamaría Rodríguez ${ }^{\pi}$
}

Artículo de investigación producto del proceso de formación doctoral en Educación de los autores en la Universidad Católica de Córdoba, Argentina.

$\S$ Doctorando en Educación de la Universidad Católica de Córdoba, Argentina. Especialista y magíster en Bioética de la Universidad del Bosque, Bogotá, Colombia. Licenciado en Teología de la Pontificia Universidad Javeriana, Bogotá, Colombia. Docente tiempo completo Facultad de Educación de la Universidad Santo Tomás.

Correo electrónico:

johnperezv@ustadistancia.edu.co

(iD) 0000-0001-9978-3997

‡ Doctorando en Educación de la Universidad Católica de Córdoba, Argentina. Magíster en Educación de la Universidad Santo Tomás, Bogotá, Colombia. Licenciado en Filosofía y Educación Religiosa de la Universidad Santo Tomás, Bogotá, Colombia. Docente tiempo completo Facultad de Educación de la Universidad Santo Tomás.

Correo electrónico:

johannieto@ustadistancia.edu.co

(iD) 0000-0002-8608-8511

" Doctorando en Educación de la Universidad Católica de Córdoba (Argentina). Magíster y licenciado en Teología de la Pontificia Universidad Javeriana. Especialista en Pedagogía para la Educación Superior de la Universidad Santo Tomás (Colombia). Docente e investigador en la Facultad de Teología de la Universidad Santo Tomás (Colombia).

Correo electrónico:

juanessantrax87@gmail.com

(iD) $0000-0002-4632-4700$

\section{Cómo citar:}

Pérez, J., Nieto-Bravo, J., y Santamaría-Rodríguez, J. (2019). La hermenéutica y la fenomenología en la investigación en ciencias humanas y sociales. Civilizar: Ciencias Sociales y Humanas, 19(37), 21-30. doi: 10.22518/usergioa/jour/ccsh/2019.2/ aog

\section{Resumen}

Este artículo de reflexión problematiza las relaciones entre la fenomenología y la hermenéutica como lugares epistémicos del quehacer investigativo por medio de un abordaje documental construido en dos fases. En primer lugar, el texto reflexiona alrededor del uso terminológico presente en los paradigmas, enfoques, perspectivas epistemológicas y métodos investigativos, identificando con ello que no existe unicidad de criterios en sus referencias y aproximaciones en los tratados de metodología de la investigación analizados. De igual manera, se evidencian que hay propuestas metodológicas que comprenden la fenomenología y la hermenéutica de manera complementaria, articulada o aislada sin permitir con ello lugares precisos de comprensión que permitan situar su aplicación en la investigación. En razón de lo anterior, la segunda fase propone una aproximación individual a los antecedentes y comprensiones de la fenomenología y la hermenéutica, identificado particularidades que les caracterizan, posibles diferencias teórico-prácticas y aproximaciones que se pueden establecer de cara a su relevancia desde el marco epistémico y metodológico de la investigación en las ciencias humanas y sociales.

\section{Palabras clave}

Hermenéutica; fenomenología; investigación; ciencias humanas y sociales, metodología de la investigación.

\section{Abstract}

This article of reflection problematizes the relationships between phenomenology and hermeneutics as epistemic places of research work through a documentary approach built in two phases. First, the text reflects on the terminological use present in the paradigms, approaches, epistemological perspectives and research methods, thereby identifying that there is no unique criteria in its references and approaches in the research methodology treaties analyzed. In the same way, it is evident that there are methodological proposals that include phenomenology and hermeneutics in a complementary, articulated or isolated way without allowing precise places of understanding that allow placing their application in research. Based on the foregoing, the second phase proposes an individual approach to the background and understandings of phenomenology and hermeneutics, identifying particularities that characterize them, possible theoretical-practical differences and approaches that can be established for their relevance from the framework Epistemic and methodological research in social human sciences.

\section{Keywords}

Hermeneutics, phenomenology, research, human and social sciences, research methodology. 


\section{Introducción}

Los procesos de investigación en ciencias humanas y sociales exigen a sus investigadores vérselas con conceptos como la fenomenología y la hermenéutica. Ambos son empleados con frecuencia en el campo de la metódica investigativa de orden cualitativo como perspectiva epistemológica que sitúa el lugar de enunciación de las pesquisas en estos campos de conocimiento. Desde sus orígenes, el aporte a la comprensión de los fenómenos o hechos de carácter humano y social ha sido incuantificable, constituyéndose así en herramientas recurrentes a las que acuden las y los investigadores y/o grupos de investigación para sentar las bases de sus indagaciones y procesos.

Aunque su uso es frecuente, y en algunos casos necesario y pertinente, hay tensiones en su apropiación a nivel epistemológico y metodológico. Estos elementos constituyen una primera tensión en relación a su papel y lugar en la investigación pues no resulta ser unívoco de conformidad con varios planteamientos de autores o manuales de la investigación. En un segundo momento, aquellos exigen el reconocimiento de sus implicaciones, características, impactos o particularidades. Todo ello, a fin de poder decantar las alternativas que ofrecen a la investigación social desde su episteme y metódica.

El presente artículo indaga alrededor de la fenomenología y la hermenéutica para identificar sus aportes y usos en el campo de la investigación de carácter humano y social. Al reconocer sus particularidades, implicaciones y posibilidades de articulación o de trabajo independiente, esta pesquisa abre caminos para aproximarse a sus horizontes de comprensión, que serán un aporte sustancial para clarificar su relevancia en la investigación humana y social en correlación a las especificidades de los proyectos investigativos que emergen en lo cotidiano.

Sobre estos argumentos, se propone una revisión de carácter documental que acude a la indagación a partir de las propuestas emanadas de textos de algunos representantes de la metodología en la investigación con el fin de sondear y reconocer ciertas tensiones alrededor de los abordajes epistémicos y metodológicos en el campo de la fenomenología y la hermenéutica. A partir de su reconocimiento, se profundiza en la materia con algunos otros textos de corte metodológico y epistemológico, que contribuyen al análisis y reconocimiento de sus particularidades y relacionalidades.
El documento está integrado por un primer apartado que aborda de manera indagatoria la temática al reconocer el lugar que ocupan estas áreas en el plano de la metodología de la investigación. En ello describe a nivel general algunas tensiones y posibilidades en sus usos. Posteriormente, la pesquisa profundiza en la comprensión de la fenomenología y de la hermenéutica en relación con sus epistemologías y usos metodológicos, señalando algunas de sus particularidades, semejanzas, diferencias y/o posibles articulaciones.

\section{El uso de la hermenéutica y la fenomenología en la investigación}

Al hablar de investigación en ciencias humanas y sociales es común encontrar en algún punto de la discusión metodológica referencias a la fenomenología y la hermenéutica, bien sea para hacer uso de las mismas como enfoque o perspectiva epistemológica, o para conocerles y abordar otra opción. De esta manera, puede afirmarse que su presencia es constante en la construcción de los sustentos metodológicos al interior de la investigación en este campo de conocimiento, marcando con ello, orientaciones teórico-prácticas puntuales por la episteme que de ellas subyace.

Tanto la fenomenología como la hermenéutica, en el ámbito de la investigación en ciencias humanas y sociales, son abordadas de distintas maneras: enfoques, paradigmas y métodos, entre otros. Con ello, una problemática en este lugar de la investigación, de su uso y referencias es que el espacio que allí ocupan no es del todo preciso, pues depende de la comprensión que las y los investigadores hagan de las mismas, pues en la documentación metodológica al respecto no hay una unidad de criterios y comprensiones, dificultando las aproximaciones a la hermenéutica y la fenomenología en el campo investigativo.

En coherencia con lo anterior, a continuación, y de manera breve, se proponen cuatro muestras de algunas recurrencias por parte de autores seleccionados por su producción académica en el campo de la metodología de la investigación. Esto, con el fin de presentar, conocer, dimensionar y problematizar estas disyuntivas acerca del uso de la fenomenología y la hermenéutica en el campo de la investigación en ciencias humanas y sociales; y con base en ello, poder abrir el paso a la comprensión de sus alcances e implicaciones en la investigación de este tipo. 
$\mathrm{Al}$ interior de su obra, José Marín (2006) inscribe la fenomenología y la hermenéutica dentro del campo de los enfoques, entendidos como una acción o un efecto de ubicar un objeto en un punto preciso. Para este autor, los enfoques pueden ser analíticos o sistémicos. Los primeros se caracterizan porque se centran en las partes; los segundos, en la totalidad de los fenómenos, eventos y objetos, entre otros. En ellos sitúa a la fenomenología y la hermenéutica en un macro-contexto de enfoques investigativos en ciencias humanas y sociales integrado también por los enfoques históricos, socio-críticos y de los sistemas complejos.

Por otra parte, Pablo Páramo (2011) sitúa la fenomenología y la hermenéutica en el ámbito de las posturas epistemológicas o paradigmas, entendiendo estos como "el conjunto de suposiciones de carácter filosófico de las que se valen los investigadores, la mayor parte de las veces de forma tácita, para aproximarse a la búsqueda de conocimiento" (p. 22). Con esta descripción, los usos epistémicos y metodológicos de la fenomenología o de la hermenéutica son relevantes en la labor investigativa y ha de configurar el rumbo de toda la investigación de acuerdo con sus particularidades.

Por su parte, Alicia Gurdián (2007) y Bolaños (2015) sitúan la fenomenología y la hermenéutica a nivel de un método. Esto implica que su uso ha de tener una clara intencionalidad, un orden y una sistematicidad que, a la postre, desemboca en procedimientos y resultados medibles que soportan el quehacer investigativo.

Con base en lo propuesto por estos autores, es latente la disyuntiva a la que se somete la comprensión de uso de la fenomenología y la hermenéutica en el ámbito investigativo. Según se aprecia, no hay un posicionamiento único, claro y definido en el campo de la investigación, lo que, por ejemplo, hace que la concepción o fundamentación de cursos y panoramas investigativos claves para las elaboraciones metodológicas de las pesquisas en ciencias humanas y sociales entren en una constante disruptiva conceptual y epistémica. En esta línea, Pablo Páramo (2011) llama la atención al afirmar que:

El estudio sobre la evolución reciente de las distintas posturas epistemológicas permite evidenciar que no se pretende unificar en un paradigma único los distintos discursos epistemológicos, no se busca un discurso homogéneo y hegemónico sobre la manera de construir el conocimiento, lo cual era más propio de la modernidad, aunque sí es posible adoptar unas reglas básicas sobre las distintas maneras de llevar a cabo la investigación como que las teorías han de ser coherentes, lógicamente firmes y corresponder con los datos de la observación obtenidos de modo objetivo y ser procesados de manera adecuada. (p. 29).

Sumado a esta proposición de Páramo (2011), es importante reconocer que la investigación en ciencias humanas y sociales no cuenta con una fórmula de carácter algorítmico, que conlleve a una fundamentación o sentido epistemológico o metodológico único y preciso que permita establecer unicidad de teorías conducentes a planteamientos metodológicos. Las herramientas de las y los investigadores son múltiples y así sus usos. Por ello, la constitución como método para un autor o investigador no es lo mismo que para otro, lo mismo sucede con los enfoques, las perspectivas y los paradigmas, entre otros aspectos que circundan el panorama metodológico (Rivadeneira, 2015).

De esta manera, aplicado al tema de la fenomenología y la hermenéutica, independientemente de que se reconozcan como enfoques, como paradigmas o como métodos, es importante considerar su sentido, las implicaciones que tiene al interior de la investigación y el lugar que las y los investigadores le determinen; teniendo presente para ello, los alcances y posibilidades que ofrecen al interior de las metodologías por los cuales queda garantizada la pertinencia y la coherencia en el quehacer investigativo.

Con el abordaje de estas comprensiones a nivel de metodología de la investigación se da paso a profundizar en los alcances de la fenomenología y de la hermenéutica. Este aspecto no es una cuestión menor ya que desde sus orígenes y desarrollos posteriores se han visto íntimamente relacionadas a la vez que varias propuestas de metodólogos conciben su uso independiente.

La posibilidad de articulación o de independencia que se debate entre la naturaleza epistemológica de la fenomenología y la hermenéutica tiene en la base las orientaciones metodológicas que analizan el mundo y su lugar referido a nivel epistémico en los procesos de investigación. Es por esta razón que a continuación se propone una breve aproximación alrededor de ambas a fin de analizar sus orígenes, propuestas, límites y posibilidades de articulación. 
A partir de este análisis, también se pretende caracterizar sus rasgos identitarios, permitiéndole con ello a las y los investigadores tener puntos de referencia al momento de determinar el lugar epistémico y metodológico de sus pesquisas, así como también sus posibilidades de articulación o de trabajo independiente.

\section{La fenomenología como escenario epistemológico del quehacer investigativo}

En la metódica investigativa, es relevante identificar una doble discusión de sentido en donde se confronta la intencionalidad nomotética de explicación y la perspectiva ideográfica de interpretación y descripción (Wright, 1979). El monismo metodológico que marcó las formas de hacer investigación consideró que la enunciación de leyes mecánicas expresadas en el lenguaje universal de la física y la matemática era la única vía posible para generar conocimiento (Comte, 1984). Frente a esto, los movimientos ideográficos motivados por la fenomenología husserliana y la hermenéutica se comprometieron a efectuar un acercamiento a la realidad, capaz de trascender la intencionalidad explicativa (Erklären) de las ciencias exactas, caracterizadas por la generalización de los postulados que han transitado por el método hipotético-deductivo, para aterrizar en la interpretación como plataforma de comprensión (Verstegen) que subjetiviza y particulariza al fenómeno (Droysen, 1983).

Sobre este presupuesto, es necesario leer la fenomenología como lugar epistemológico de la investigación. Ello toma una distancia de la teorización especulativa nomotética para recibir y describir aquello que se manifiesta a la conciencia tal cual es (Husserl, 1992). Dicho giro epistémico supera la materialización de lo medible para abrir la puerta a aquello que, naciendo de lo sensible, es problematizado en la conciencia (Husserl, 1994).

La conciencia devela el rostro de la subjetividad que ha sido eclipsado por el objetivismo positivo, trascendiendo la hipótesis explicativa para transitar por la "epojé" como riqueza de la investigación cualitativa, que pone en sospecha - Husserl (1992) los describe con la imagen de poner entre paréntesis - los pre-saberes eruditos, con el fin de abrirse a la posibilidad de la quaestio como forma de problematización de la realidad, tal como se presenta, que parte del indagar y se compromete con buscar la verdad (Parra, 2011).
Un aporte relevante de este lugar epistémico a las formas de investigar con enfoques cualitativos es el retorno a la subjetividad, pues dentro de la construcción empírico-analítica del conocimiento positivo, siempre se vio como un riesgo visibilizar y escuchar la voz de los sujetos, ya que podría tambalear la exactitud que ofrece lo objetivo, propendiendo así el error. Sin embargo, esto no se puede constituir en una forma de solipsismo en el que el sujeto se vuelca sobre sí y no es capaz de dialogar con el otro. Este es quizás el principal talón de Aquiles de la fenomenología de Husserl (1992), pues redujo la intersubjetividad al campo de la conciencia. En la actualidad muchos investigadores cualitativos siguen transitando por esta vía, impidiendo el encuentro entre la fenomenología y la acción social.

El filósofo alemán Alfred Schütz (1993) propone dar un giro fenomenológico al volcar este estudio a la sociedad, al trascender el ámbito de la conciencia y darle una dimensión social cuyo epicentro es el fenómeno de lo intersubjetivo. Este no responde al fuero de lo privado o a una mentalidad intimista, más bien se abre a la dimensión del encuentro dialógico con el otro. De allí que una investigación fenomenológica bucea en la conciencia del ego, pero también discute en la experiencia del alter.

Una fenomenología social puede fundamentar el quehacer del investigador cualitativo, cuya preocupación es consolidar un conocimiento incluyente mediado por la experiencia de intersubjetividad. Aquella se da en "en el presente vivido, en el cual hablamos y nos escuchamos unos a otros" (Mieles, Tonon y Alvarado, 2012, p. 208), donde la realidad del cotidiano constituye el tejido colectivo de la conciencia social y los sujetos comparten la vida como lugar de interconexión en el que fluye la conciencia.

La revolución contemporánea del saber ha motivado las transformaciones de la sociedad actual, la cual está atravesada por una triada integrada por la vida cotidiana, la ciencia y la reflexividad técnica (Valera, 2008). Así las cosas, la construcción del conocimiento no se da unilateralmente por la comprobación o falsación de hipótesis, sino más bien, "por la maduración de problemas que no encuentran solución a menos que se reconstruyan o se sustituyan dicotomías cognoscitivas y viejos ideales" (Sotolongo y Delgado, 2006, p. 28), en últimas, por la capacidad de poner entre paréntesis.

Investigar desde una perspectiva fenomenológica implica despejar la indagación de las 
macro-comprensiones creadas por el artificio teórico, "para designar la apariencia de las cosas sensibles como contrapuestas a su ser esencial o inteligible" (Marín, 2006, p. 127). En consonancia con el pensamiento kantiano, "la expresión "fenómeno" se opone a la expresión "cosa en sí misma”, [la cual] es una representación de nuestra sensibilidad. Todo objeto de una intuición empírica es fenómeno" (Caimi, 2017, pp. 201-202). Esta noción se opone al noúmeno al trascender lo ininteligible desde la perspectiva de la sensibilidad humana, motivo por el cual este "se muestra por sí mismo, haciéndose manifiesto y visible" (Castillo, 2000, p. 29) al conocimiento humano.

La consolidación de la fenomenología como un campo desde el cual se puede construir conocimiento investigativo ha propiciado procesos de tránsito que continúan cambiando, por ello no puede ser concebida "sólo como un método de descripción esencial de las articulaciones fundamentales de la experiencia (perceptiva, imaginativa, intelectiva, volitiva, axiológica, etc.), sino como una auto-fundamentación radical en la más completa claridad intelectual" (Ricoeur, 2000, pp. 200-201).

José Tadeo (2011) complementa esta apreciación proponiendo que la fenomenología se comprende como ciencia proveniente de la filosofía, ciencia que se fundamenta en la comprensión de los fenómenos cuya debilidad es la ausencia de un método sobre el cual se pueda filosofar. De ahí que muchas veces nace la necesidad de complementarla con la hermenéutica. No obstante, lo anterior, a este autor se le puede criticar la noción de ciencia que subyace en este contexto. La ciencia es el resultado de un modo o manera de conocer, en cambio, la filosofía se inscribe más en el ámbito críticoreflexivo que se ubica en un nivel discursivo; por lo tanto, una cosa resulta ser el conocimiento científico y otra el conocimiento filosófico.

Identificar la plataforma desde la cual se investiga cualitativamente con una episteme fenomenológica propicia la no presuposición de los resultados o hallazgos de la investigación. Esta no ha de estar antecedida, por ejemplo, por el sentido común, propuestas científicas, experiencias psicológicas, creencias y/o prejuicios. De ahí que la fenomenología, en palabras de Gurdián (2007):
No parte del diseño de una teoría, sino del mundo conocido, del cual hace un análisis descriptivo con base en las experiencias compartidas, en donde el mundo conocido y de las experiencias intersubjetivas [ofrecen] las señales o avisos para interpretar la diversidad de símbolos. (pp. 151-152).

La postura defendida por Gurdián (2007) visibiliza los sujetos y las subjetividades que se constituyen en parte esencial de los abordajes metodológicos. Según Martínez (2011), la fenomenología "se preocupa por la comprensión de los actores sociales y por ello de la realidad subjetiva, comprende los fenómenos a partir del sentido que adquieren las cosas para los actores sociales en el marco de su proyecto de mundo" (p. 18). Por ello, los individuos son parte fundamental de la comprensión fenomenológica al asumir el rol de cognoscentes, los cuales dotan de sentido las experiencias vividas sobre el fenómeno y están en la capacidad de poder describirle (Creswel, 1998).

Bajo estos presupuestos, se comprende que la fenomenología "no está en última instancia interesada en la explicación. La cuestión típica formulada no es ‘qué causa X?', sino ‘qué es X?'[...] La investigación fenomenológica enfatiza los aspectos individuales y subjetivos de la experiencia" (Sandín, 2003, p. 16). Así pues, "se puede afirmar que un presupuesto epistemológico y ontológico de la fenomenología consiste en que el ser se manifiesta, se expone al mundo de la vida" (Bolaños, 2015, p. 42), siendo este mundo de la vida el lugar en el cual se desarrollan los fenómenos y, por ende, el escenario por excelencia de conocimiento e interpretación de los mismos.

Los procesos de investigación pensados desde la fenomenología dotan de sentido a la comunidad y a la experiencia vivida frente al fenómeno. Son aspectos constitutivos de estos procesos de indagación la naturaleza descriptiva que propicia la oportunidad de estudio, análisis y reflexión sobre el fenómeno. De igual manera, es importante reconocer un rigor y un carácter académico que propicia trascender el plano de las descripciones subjetivas, para lograr un alcance o una intencionalidad de comprensión intersubjetiva, posibilitando de esta manera los dinamismos del conocimiento científico y su uso en los procesos de investigación.

Puede entonces identificarse que uno de los grandes retos en la producción científica es la consolidación de formas alternativas de construir cono- 
cimiento. Ritzer (1998) lo denomina la integración o síntesis de paradigmas, hecho que reconoce que las fronteras epistemológicas de la investigación son conurbadas. Por ello se requiere de una interacción relacional desde el campo de lo cualitativo, donde los fenómenos se encuentren cara a cara como forma de constitución de la conciencia y del tejido social (Ritzer, 1998).

El giro epistémico actual traza el reto de transitar entre los paradigmas que simplifican a los que complejizan, es decir, que la fenomenología puede avanzar de la observación del objeto a la del fenómeno y la red sistémica que lo observa y determina (Espina, 2003). La figura de la interrelación fenoménica es "como un acto de revelación en el que un ser humano se manifiesta, se hace presente ante otro para enseñarle, para mostrarle algo de su propio ser, para ayudarlo a descubrirse a sí mismo" (Ferreyra y Blanas, 2011, p. 18), hecho que se constituye en proyecto de crecimiento para la sociedad del conocimiento que, a pesar de avanzar tecnológicamente, sigue encontrando relaciones humanas fracturadas (Ferreyra y Caelles, 2010).

Se puede concluir que el "actuar humano está abierto a las diversas interpretaciones que se generan gracias a la comprensión que se realice de este" (Nieto, 2017, p. 177). Lo anterior basta para señalar que los lugares epistemológicos desde donde se investiga no son fragmentos atomizados de una realidad, sino escenario de interrelaciones del conocimiento cuyas fronteras son delgadas; tanto que solo se pueden reconocer tras las intencionalidades investigativas que responden a la necesidad del contexto.

\section{La hermenéutica como escenario epistemológico del quehacer investigativo}

Una vez establecidas ciertas apreciaciones alrededor de la fenomenología, es momento de explorar el papel y lugar de la hermenéutica en los procesos investigativos. Para este propósito se expone una definición genérica al interior de sus sustentos epistemológicos y de la historia, develando con ello, el paso y los alcances que tiene para los desarrollos investigativos de las ciencias humanas y sociales en la actualidad.

La hermenéutica tiene sus raíces etimológicas en la palabra griega hermeneutikos que, a modo general y reductivo, se relaciona con el arte de interpretar. En sus orígenes griegos, este término se aso- cia a la figura de Hermes, uno de los hijos de Zeus, y quien asumía la tarea de llevar a los seres humanos los mensajes de los dioses, velando por que puedan ser comprendidos. De ahí que la hermenéutica esté asociada al arte de interpretar o comprender textos escritos o realidades, haciendo del texto algo rebasa la escritura (Ricoeur, 1990).

El origen de la hermenéutica no puede estar desligado de la fenomenología según establece Gurdián (2007). Es en este punto en donde se encuentra la mayor dificultad para diferenciar estas dos perspectivas, pues al tener un germen en común, es una tarea delicada y detallada la que se requiere para establecer sus límites y fronteras. Así pues, para definir estas últimas es menester profundizar en los sustentos epistemológicos de la hermenéutica, y con ello, se abre la posibilidad de poder trazar de manera precisa, o al menos cercana, los límites y relaciones entre fenomenología y hermenéutica aplicadas al campo de la investigación en ciencias humanas y sociales.

En su recorrido histórico hasta el siglo XVIII, Marín (2006) aclara que "la hermenéutica estaba relacionada con la comprensión e interpretación de textos sagrados, pero con el desarrollo del racionalismo y de la filología clásica comienza propiamente la historia de la hermenéutica en su acepción moderna" (p. 132). En este sentido, no se puede desconocer que la hermenéutica se consolida con el pensamiento occidental (crítica literaria, filosofía y ciencias sociales) y, de especial manera, con el interés de las tradiciones religiosas cristianas (católica y protestante) en su apuesta por comprender el sentido de la revelación divina desde las Sagradas Escrituras y una lectura de contexto de la misma desde la experiencia de fe (Noratto y Suárez, 2007, pp. 113-121).

Ahora bien, siendo la hermenéutica eje epistémico y metodológico de la ciencia teológica, no es exclusiva de ella. Es importante destacar su apropiación en diversas áreas de las ciencias humanas y sociales, debido a que sus fundamentos epistemológicos ofrecen elementos esenciales para comprender objetos, símbolos, textos y realidades, entre otros aspectos, en contraste con las tensiones epistémicas surgidas entre los siglos XVIII y XIX acerca de la pretensión de verdad y validez del conocimiento entre las ciencias del espíritu y las positivas (monismo metodológico). Sobre esta coyuntura, Mardones (2005) afirma: 
De todo este esfuerzo por precisar una filosofía de la ciencia que no caiga en las redes del positivismo y haga justicia a la peculiaridad de las ciencias humanas, culturales o del espíritu, una cosa queda clara: el rechazo a aceptar el modelo de explicación científica triunfante en Occidente desde Galileo. Hay una recuperación de la tradición aristotélica, a través de Hegel. (p. 32).

De manera general y en relación con su raíz en la fenomenología de Husserl, "la hermenéutica intenta establecer un proceso por medio del cual se haga, inicialmente, una interpretación en torno al sentido de cualquier fenómeno, y en una segunda instancia, se realice la comprensión del mismo" (Gutiérrez, 1986, p. 57). Esta acepción posibilita la distinción de la fenomenología en relación con la hermenéutica, en la cual se pueden distinguir dos claros niveles de esta última: interpretación y comprensión, que según afirma Mardones (2005), toman distancia del paradigma positivista de la ciencia y su absolutización filosófica como única vía para la construcción de conocimiento y explicación de la realidad.

Autores como "Heidegger y Gadamer definieron la hermenéutica como la autocompresión, la cual no sería otra cosa que la comprensión del propio ser como ser en el mundo" (Herrera, 2003, p. 1). De tal manera que la hermenéutica se relaciona a un profundo proceso reflexivo, de conocimiento e interpretación que se distingue de la fenomenología en tanto que esta última se comprende de una manera más analítica y comprensiva del mundo y los diversos sentidos.

Según Ricoeur (2000), la hermenéutica se mantuvo en el plano de los textos por mucho tiempo, restringiendo su uso a los mismos y vetando o descuidando otras esferas que pueden ser objeto de interpretación. Esto supuso su detrimento al no ser reconocida, por ejemplo, desde las tradiciones orales que son parte esencial en la configuración de las comunidades. En esta línea, el autor se permite hacer una alusión al discurso como una reivindicación de aquellas.

Gracias a la escritura, el discurso adquiere una triple autonomía semántica: respecto a la intención del locutor, a la recepción del auditorio primitivo y a las circunstancias económicas, sociales y culturales de su producción. En este sentido, lo escrito se aleja de los límites del diálogo cara a cara y se convierte en la condición del devenir-texto del discurso. Corresponde a la hermenéutica explorar las implicaciones que tiene este devenir-texto para la tarea interpretativa (Ricoeur, 2000, p. 204).

Como complemento de lo anterior, Martínez (2011) integra el valor de la comunidad y el papel de la hermenéutica en este sentido, pues la misma "busca descubrir los significados de las distintas expresiones humanas, como las palabras, los textos, los gestos, pero conservando su singularidad" (p. 17), de tal manera que se articula en su labor la realidad en un sentido mucho más amplio y no se limita a los textos exclusivamente. Al respecto, Gurdián (2007) llama la atención sobre lo siguiente:

Recordemos que la hermenéutica es un método general de comprensión y la interpretación es el modo natural de conocer de los seres humanos. La hermenéutica tiene como misión descubrir los significados de las cosas, interpretar lo mejor posible las palabras, los escritos, los textos y los gestos, así como cualquier acto y obra, pero conservando su singularidad en el contexto del que forma parte (p. 146).

De esta manera, y aplicado al plano de la investigación en las ciencias humanas y sociales, se ha de reconocer en la hermenéutica un gran valor dado su papel en la profunda interpretación del mundo de la vida y sus componentes por parte de los sujetos. Estas acepciones plantean una tarea doble: "reconstruir la dinámica interna del texto (objeto, situación, símbolo, realidad, entre otros) y restituir la capacidad de la obra para proyectarse al exterior mediante la representación de un mundo habitable" (Ricoeur, 2000, p. 205). Es en ello en donde "el ejercicio hermenéutico ha estado asociado a la sutileza o agudeza (...), las cuales vienen a constituir la esencia propia de la hermenéutica" (Noratto y Suárez, 2007, p. 121). Esto es, a su realización desde el sujeto en su realidad histórica, en donde tales aspectos dan razón de la hermenéutica desde tres formas implicativas para quien interpreta, a saber:

- Subtilitas intelligendi o capacidad para entender aquello que está siendo leído o interpretado en el mundo de la vida (texto).

- Subtilitas explicandi o ampliación del significado del texto o de la realidad desde el proceso de apropiación que realiza el sujeto (contexto).

- Subtilitas applicandi o incorporación al mundo y/o realidad apropiada por parte del sujeto intérprete (hermeneuta) con una 
salvedad en cuanto a su distancia y la configuración de su sentido (pretexto).

Así las cosas, la hermenéutica se constituye como un elemento esencial en la labor investigativa a la luz de la implicación vital de quien la realiza. Particularmente, asume una triple dinámica (texto, contexto, pretexto) porque a la tarea de la interpretación es necesaria una apropiación de la realidad investigada sobre la cual sea posible comprender aquello que representa (texto), el lugar en el cual está situada (contexto) y su intencionalidad y/o devenir (pretexto).

$\mathrm{Su}$ aplicación a nivel de la investigación en ciencias humanas y sociales ha de suponer un trabajo serio, metódico y profundo que supera el plano descriptivo o valorativo, adentrándose en la cosa en sí misma para traer a la luz sus reales significados para los intérpretes, al interior de sus contextos y redes simbólicas, lo que permite con ello una nueva visión, por demás, crítica, objetiva y diferenciada de la realidad aparente. En ello radica su diferencia con la fenomenología, pues al interés descriptivo de esta, la hermenéutica tiene por finalidad una apropiación de la realidad y/o de la textualidad como condición de posibilidad para comprenderla en su estructura, realidad e intencionalidad, sin dejar de estimar los conflictos que puedan surgir de las mismas interpretaciones, sean estas desde la objetividad y/o desde la subjetividad de quien la realiza (Ricoeur, 2003).

\section{Conclusiones}

En conformidad con varios metodólogos o textos de metodología de la investigación, se puede inferir que el uso de la fenomenología y la hermenéutica no es unívoco, pues su lugar lo otorga la comprensión de investigación que subyace, la autonomía de las y los investigadores que los emplean o su implementación. En esta medida es común encontrar que algunos investigadores las refieren como paradigmas, enfoques, perspectivas, tipos de investigación, entre otros.

A nivel epistemológico se reconocen algunos rasgos que caracterizan las particularidades de la fenomenología y de la hermenéutica y su aplicación en el ámbito de la metódica de la investigación en las ciencias humanas y sociales. En reconocimiento de lo anterior, y a modo de síntesis, se propone una breve descripción de algunas de las relaciones, diferencias, similitudes y alcances, entre otras posibilidades, que marcan estas dos corrientes y permiten dimensionar su papel en los contextos investigativos.
En primer lugar, es perceptible el origen en común que comparten la hermenéutica y la fenomenología. La primera deriva de la segunda en los desarrollos históricos a partir de Husserl (1992; 1994), no obstante, a pesar de su origen en común, las dos alcanzan dimensiones de aplicación, reflexión y de investigación diferentes.

La fenomenología se caracteriza por su abordaje y lugar de acción sobre los fenómenos, o lo que es similar, se presenta ante la realidad ejerciendo un papel descriptivo. De ahí que le otorgue sentido al mundo que se vive. Por su parte, la hermenéutica profundiza mucho más en los sentidos y la búsqueda fundamentada de lo que es en sí, es decir se juega en una dimensión mucho más ontológica.

En un segundo momento, es común encontrar que la hermenéutica ha alcanzado su esplendor basando su trabajo en la interpretación de textos, dada la naturaleza de los mismos y su exposición en el mundo. En este sentido, la labor del hermeneuta está situada en determinar o identificar la intencionalidad original del texto para poder transmitirla públicamente. Es común apelar a la hermenéutica en las investigaciones de carácter documental, no obstante, su uso no se puede limitar a estos escenarios, logrando ser un excelente complemento en otras estancias investigativas vinculadas a las oralidades, narrativas y construcciones de memoria social, histórica y/o colectiva.

Finalmente, sobre el interés de distinguir ambos estrados, Gurdián (2007) propone la siguiente diferenciación al hablar de la hermenéutica y de la fenomenología:

a) El método hermenéutico trata de introducirse en el contenido y la dinámica de la persona estudiada, en sus implicaciones y busca estructurar una interpretación coherente del todo.

b) El método fenomenológico respeta, por completo, el relato que hace la persona de sus propias vivencias. Se centra en el estudio de las realidades vividas o vivencias, generalmente, poco comunicables.

Junto a lo anterior, es válido aclarar que, pese a las diferencias establecidas, también se puede hablar de una complementariedad que soporta la investigación. Es decir, que el uso de la hermenéutica o la fenomenología en la investigación no es univoco, o unidireccional, de tal manera que su aplicación demanda una continua actitud de descubrimiento, conocimiento e interpelación que conduzca a las reflexiones epistemológicas (Tadeo, 2011). 


\section{Referencias}

Bolaños, R.(2015).Elementosdehermenéuticayfenomenologíaparaundiálogometodológicoentrelasciencias. Revista Sophia, 19, 25-46. doi: 10.17163/soph. n19.2015.01

Caimi, M. (coord.) (2017) Diccionario de la filosofía crítica kantiana. Ciudad Autónoma de Buenos Aires, Argentina: Colihue.

Castillo, E. (2000). La fenomenología interpretativa como alternativa apropiada para estudiar los fenómenos humanos. Revista en Educación y Enfermería, $18(1), 27-35$.

Comte, A. (1984). Discurso del espíritu positivo (2. ${ }^{\mathrm{a}} \mathrm{Ed}$.). Madrid, España: Alianza Editorial.

Droysen, J. G. (1983). Histórica: lecciones sobre la enciclopedia y metodología de la historia. Barcelona, España: Alfa.

Espina, M. (2003). Complejidad y pensamiento social. En L. Carrizo, M. Espina, J. Klein. Transdisciplinariedad y complejidad en el análisis social. París, Francia: UNESCO

Ferreyra, H., y Caelles, S. (2010). Más allá del paradigma del consenso y del conflicto: el modelo del encuentro educativo. En H. Ferreyra (Ed.), Actualidad y campo pedagógico. Textos con el pretexto de habilitar el debate. Bogotá, Colombia: USTA. doi: 10.15332/s2011-8643.2009.0005.02

Ferreyra, H., y Blanas, G. (2011). Introducción. En O. Parra, El Placer de conocer investigando. (pp. 9-19). Bogotá: USTA.

Gurdián, A. (2007). El paradigma cualitativo en la investigación Socio-Educativa. Costa Rica: CECC-AECI.

Gutiérrez, G. (1986). Cap. II - Metodología de las Ciencias Sociales II (pp. 139-165). México: Harla.

Herrera, D. (2003). Fenomenología y hermenéutica. Revista de la Facultad de Artes y Humanidades, (17), 1-15.

Husserl, E. (1992). Invitación a la fenomenología. España: Paidós Ibérica.

Husserl, E. (1994). Problemas fundamentales de la fenomenología. Madrid, España: Alianza.

Mardones, J. (2005). Filosofía de las ciencias humanas y sociales. Bogotá D.C., Colombia: Editorial Anthropos.

Marín, J. (2006). La investigación en educación y pedagogía. Sus fundamentos epistemológicos y metodológicos. Bogotá, Colombia: USTA.

Martínez, J. (2011). Métodos de investigación cualitativa. Silogismos de investigación, (08), 1-43.
Mieles, M., Tonon, G., y Alvarado, S. (2012). Investigación cualitativa: el análisis temático para el tratamiento de la información desde el enfoque de la fenomenología social. Universitas humanística, 74, 195-225.

Nieto, J. A. (2017). Hacia una didáctica del sentido. Didácticas emergentes de las prácticas pedagógicas de filosofía en tres escuelas católicas. Revista Interamericana de Educación, Pedagogía y Estudios Culturales, 10(1), 173-195. doi: 10.15332/s1657107X.2017.0001.07

Noratto, J., y Suárez, G. (2007). La racionalidad hermenéutica en teología. En G. Baena, D. Martínez, V. Martínez, J. Noratto y G. Suárez (Eds.), Los métodos en teología. Bogotá, Colombia: Facultad de Teología. Pontificia Universidad Javeriana.

Páramo, P. (Comp.) (2011). La investigación en ciencias sociales: estrategias de investigación. Bogotá, Colombia: Universidad Piloto de Colombia.

Parra, O. (2011). El placer de conocer investigando. Gestión del Conocimiento. Bogotá, Colombia: USTA.

Ricoeur, P. (1990). Freud: Una interpretación de la cultu-

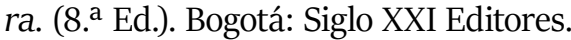

Ricoeur, P. (200o). Narratividad, fenomenología y hermenéutica. Revista Análisis, 25, 189-207.

Ricoeur, P. (2003). El conflicto de las interpretaciones. Buenos Aires, Argentina: Fondo de Cultura Económica de Argentina.

Ritzer, G. (1998). Teoría sociológica contemporánea. Madrid, España: McGraw-Hill.

Rivadeneira, E. (2015). Comprensión teórica y proceso metodológico de la investigación cualitativa. Crescendo, 6(2), 169-183. doi: 10.21895/incres.2015. v6n2.16

Sandín, E. (2003). Investigación cualitativa en educación. Fundamentos y Tradiciones. Madrid, España: Mc Graw and Hill Interamericana.

Sotolongo, P., y Delgado, C. (2006). La revolución contemporánea del saber y la complejidad social. Hacia unas ciencias sociales de nuevo tipo. Buenos Aires, Argentina: CLACSO.

Tadeo, J. (2011). Fenomenología y hermenéutica como epistemología de la investigación. Revista Paradigma, 32(2), 7-22.

Valera, R. (2008). Acercamiento a la problemática actual de los estudios sociales. Curso: Participación ciudadana y educación popular, confluencias y desafío en el espacio asociativo latinoamericano. Buenos Aires, Argentina: CLACSO. 
